\title{
COMPARISON RESULTS FOR \\ BRANCHING PROCESSES \\ IN RANDOM ENVIRONMENTS
}

\author{
FRANCO PELLEREY, ${ }^{*}$ Politecnico di Torino
}

\begin{abstract}
In this note we consider branching processes whose behavior depends on a dynamic random environment, in the sense that we assume that the offspring distributions of individuals are parametrized, over time, by the realizations of a process describing the environmental evolution. We study how the variability in time of the environment modifies the variability of total population by considering two branching processes of this kind (but subjected to different environments). We also provide conditions on the random environments in order to stochastically compare their marginal distributions in the increasing convex sense. Weaker conditions are also provided for comparisons at every fixed time of the expected values of the two populations.
\end{abstract}

Keywords: Branching process; increasing convex order; supermodular order; concordance order

2000 Mathematics Subject Classification: Primary 60E15; 60G99

\section{Preliminaries and utility notions}

Branching processes are commonly used in applied probability to model the development of populations whose members produce offspring according to stochastic laws (see Harris (1989)). Initially introduced as a tool for specific biological problems, today the range of applications of branching processes includes molecular and cellular biology, human evolution, medicine, physics, computer science, and actuarial science (see Rolski et al. (1999), Teich and Saleh (2000), and Kimmel and Axelrod (2002), among others).

In the literature the classical definition of a standard branching process is the following. A branching process is a process $\boldsymbol{Z}=\left\{Z_{n}, n \in \mathbb{N}\right\}$ such that $Z_{0}$ has a known fixed distribution and

$$
Z_{n}=\sum_{j=1}^{Z_{n-1}} X_{j, n}, \quad n \geq 1 .
$$

The integer-valued random variables $X_{j, n}$, with $j, n \in \mathbb{N}$, are usually assumed to be all independent and identically distributed (i.i.d.) for every fixed $n$. Typically, the value $Z_{n}$ denotes the size of a population at the $n$th generation (or season), while the random variable $X_{j, n}$ represents the number of offspring of the $j$ th individual at the $n$th generation, with $j, n \in \mathbb{N}$. The assumption that the $X_{j, n}$ are i.i.d. means that individuals reproduce independently of each other according to some given offspring distribution.

In the literature there are several results about stochastic comparisons for population sizes of branching processes in the case where the numbers of offspring are independent. In order

Received 25 May 2006; revision received 26 September 2006.

* Postal address: Dipartimento di Matematica, Politecnico di Torino, corso Duca Degli Abruzzi 24, 10129 Torino, Italy. Email address: franco.pellerey@ polito.it 
to state two of them, we recall the definition of two well-known stochastic orders (see Shaked and Shanthikumar (1994) for properties and applications of these orders).

Definition 1.1. Let $X$ and $Y$ be nonnegative random variables. Now, $X$ is said to be smaller than $Y$ in the usual stochastic order (denoted by $X \leq_{\text {st }} Y$ ) if $\mathrm{E}[u(X)] \leq \mathrm{E}[u(Y)]$ for all increasing functions $u$ for which the expectations exist, and $X$ is said to be smaller than $Y$ in the increasing convex order (denoted by $X \leq_{\mathrm{icx}} Y$ ) if $\mathrm{E}[u(X)] \leq \mathrm{E}[u(Y)]$ for all increasing convex functions $u$ for which the expectations exist.

Now consider two standard branching processes $Z_{1}=\left\{Z_{1, n}, n \in \mathbb{N}\right\}$ and $Z_{2}=\left\{Z_{2, n}, n \in\right.$ $\mathbb{N}$, where $Z_{1,0}=Z_{2,0}=1$ almost surely (a.s.) and

$$
Z_{i, n}=\sum_{j=1}^{Z_{i, n-1}} X_{j, n}^{i}, \quad n \geq 1, i=1,2
$$

We can prove that $Z_{1, n} \leq_{\text {st }} Z_{2, n}$ for all $n \in \mathbb{N}$ whenever $X_{j, n}^{1} \leq_{\text {st }} X_{j, n}^{2}$ for all $n \in \mathbb{N}$, and that $Z_{1, n} \leq_{\text {icx }} Z_{2, n}$ for all $n \in \mathbb{N}$ whenever $X_{j, n}^{1} \leq_{\text {icx }} X_{j, n}^{2}$ for all $n \in \mathbb{N}$. The first of these cases is easy to prove, while a proof for the second case may be found in Section 8 of Ross (1983).

In this paper, we are interested in generalizations of these results in the case that the offspring distribution of individuals depends on environmental conditions (see, e.g. Smith and Wilkinson (1969), Athreya and Karlin (1970), or Jagers and Lu (2002) for examples of applications of branching processes defined on random environments). In particular, in this paper we focus on studying how the variability in time of the environment modifies the variability of the total population.

To this end, it is possible to generalize the setup above to situations in which the distribution of the numbers of offspring depends on some random geographical or economic environment $\boldsymbol{\Theta}$. This can be modeled as follows. Let $\mathcal{X} \subseteq \mathbb{R}$, and let $\boldsymbol{\theta}=\left\{\theta_{n} \in \mathcal{X}, n \in \mathbb{N} \cup\{0\}\right\}$ be any sequence of values in $\mathcal{X}$. For each $\boldsymbol{\theta}$, let $\boldsymbol{X}(\boldsymbol{\theta})$ be an infinite array of nonnegative integer-valued random variables parametrized by $\boldsymbol{\theta}$ as follows:

$$
\boldsymbol{X}(\boldsymbol{\theta})=\left|\begin{array}{ccccc}
X_{1,0}\left(\theta_{0}\right) & X_{1,1}\left(\theta_{1}\right) & \cdots & X_{1, n}\left(\theta_{n}\right) & \cdots \\
X_{2,0}\left(\theta_{0}\right) & X_{2,1}\left(\theta_{1}\right) & \cdots & X_{2, n}\left(\theta_{n}\right) & \cdots \\
\vdots & \vdots & \ddots & \vdots & \ddots
\end{array}\right|
$$

We will assume below that, for each fixed $\boldsymbol{\theta}$, the columns of $\boldsymbol{X}(\boldsymbol{\theta})$ are independent, and that, within each column, the variables are independent. Thus, if we consider only the first $n+1$ components of $\boldsymbol{\theta}$ (i.e. if we consider the restriction $\boldsymbol{\theta}_{n}=\left(\theta_{0}, \theta_{1}, \ldots, \theta_{n}\right) \in \mathcal{X}^{n+1} \subseteq \mathbb{R}^{n+1}$ of $\boldsymbol{\theta})$ then the restriction, $\boldsymbol{X}_{n}\left(\boldsymbol{\theta}_{n}\right)$, of $\boldsymbol{X}(\boldsymbol{\theta})$ to the first $n+1$ columns is of the form

$$
\boldsymbol{X}_{n}\left(\boldsymbol{\theta}_{n}\right)=\left|\boldsymbol{X}_{0}\left(\theta_{0}\right), \boldsymbol{X}_{1}\left(\theta_{1}\right), \ldots, \boldsymbol{X}_{n}\left(\theta_{n}\right)\right|=\left|\begin{array}{cccc}
X_{1,0}\left(\theta_{0}\right) & X_{1,1}\left(\theta_{1}\right) & \cdots & X_{1, n}\left(\theta_{n}\right) \\
X_{2,0}\left(\theta_{0}\right) & X_{2,1}\left(\theta_{1}\right) & \cdots & X_{2, n}\left(\theta_{n}\right) \\
\vdots & \vdots & \ddots & \vdots
\end{array}\right|,
$$

where, given $\boldsymbol{\theta}_{n}$, the distribution of the $k$ th column of $\boldsymbol{X}_{n}\left(\boldsymbol{\theta}_{n}\right)$ depends only on $\theta_{k}, k=$ $0,1, \ldots, n$, and the variables in the column are independent. 
Now let $\boldsymbol{\theta}=\left(\theta_{0}, \theta_{1}, \ldots\right)$ be a sequence of values in $\mathcal{X}$ describing the evolution of the environment, and define, recursively, the stochastic process $\boldsymbol{Z}(\boldsymbol{\theta})=\left\{Z_{n}\left(\theta_{0}, \ldots, \theta_{n}\right), n \in \mathbb{N}\right\}$ by

$$
\begin{aligned}
Z_{0}\left(\theta_{0}\right) & =X_{1,0}\left(\theta_{0}\right), \\
Z_{n}\left(\theta_{0}, \ldots, \theta_{n}\right) & =\sum_{j=1}^{Z_{n-1}\left(\theta_{0}, \ldots, \theta_{n-1}\right)} X_{j, n}\left(\theta_{n}\right), \quad n \geq 1 .
\end{aligned}
$$

In order to consider random evolutions of the environment, we can consider a sequence $\boldsymbol{\Theta}=\left(\Theta_{0}, \Theta_{1}, \ldots\right)$ of random variables taking values in $\mathcal{X}$. Thus, we will be interested in stochastic processes $\boldsymbol{Z}(\boldsymbol{\Theta})=\left\{Z_{n}\left(\Theta_{0}, \ldots, \Theta_{n}\right), n \in \mathbb{N}\right\}$ defined by

$$
\begin{aligned}
Z_{0}\left(\Theta_{0}\right) & =X_{1,0}\left(\Theta_{0}\right), \\
Z_{n}\left(\Theta_{0}, \ldots, \Theta_{n}\right) & =\sum_{j=1}^{Z_{n-1}\left(\Theta_{0}, \ldots, \Theta_{n-1}\right)} X_{j, n}\left(\Theta_{n}\right), \quad n \geq 1,
\end{aligned}
$$

where, for every $j, k \in \mathbb{N}, X_{j, k}\left(\Theta_{k}\right)$ is a random variable such that $\left[X_{j, k}\left(\Theta_{k}\right) \mid \Theta_{k}=\theta\right]={ }_{\text {st }}$ $X_{j, k}(\theta)$. Here, ' $={ }_{\mathrm{st}}$ ' denotes equality in law.

In the case of random environments having fixed identical marginal distributions, it has been shown to be useful when dependence orders are used to compare the strength of positive dependence within two multivariate distributions (see, e.g. Joe (1997), Shaked and Shanthikumar (1997), or Bäuerle and Rieder (1997)). In this paper, we consider two of these dependence orders, whose definitions are given here. Recall that a real-valued function $\phi$ defined on $\mathbb{R}^{m}$ is said to be supermodular if $\phi(\boldsymbol{x} \vee \boldsymbol{y})+\phi(\boldsymbol{x} \wedge \boldsymbol{y}) \geq \phi(\boldsymbol{x})+\phi(\boldsymbol{y})$ for all $\boldsymbol{x}, \boldsymbol{y} \in \mathbb{R}^{m}$. Here, ' $\vee$ ' and ' $\wedge$ ' denote, respectively, the componentwise maximum and minimum.

Definition 1.2. Let $\boldsymbol{X}=\left(X_{1}, X_{2}, \ldots, X_{n}\right)$ and $\boldsymbol{Y}=\left(Y_{1}, Y_{2}, \ldots, Y_{n}\right)$ be two random vectors with equal marginal distributions. Then

(i) $\boldsymbol{X}$ is said to be smaller than $\boldsymbol{Y}$ in the supermodular order (denoted by $\boldsymbol{X} \leq \mathrm{sm} \boldsymbol{Y}$ ) if $\mathrm{E}[\phi(\boldsymbol{X})] \leq \mathrm{E}[\phi(\boldsymbol{Y})]$ for every supermodular function $\phi$ for which the expectations exist,

(ii) $\boldsymbol{X}$ is said to be smaller than $\boldsymbol{Y}$ in the concordance order (denoted by $\boldsymbol{X} \leq_{\mathrm{c}} \boldsymbol{Y}$ ) if $\mathrm{E}\left[\prod_{i=1}^{n} \phi_{i}\left(X_{i}\right)\right] \leq \mathrm{E}\left[\prod_{i=1}^{n} \phi_{i}\left(Y_{i}\right)\right]$ for every collection $\left\{\phi_{1}, \phi_{2}, \ldots, \phi_{n}\right\}$ of nonnegative and increasing functions for which the expectations exist.

We note that the supermodular order implies the concordance order (which is also called positive quadrant dependence order), while (except in the case $n=2$ ) the reversed implication does not hold (see Müller and Scarsini (2000)), and both comparisons are interpreted in the sense of $\boldsymbol{Y}$ being more positively dependent than $\boldsymbol{X}$.

We also recall the definition of the usual stochastic order in the multivariate setting, and an equivalent condition that will be used in Section 2 .

Definition 1.3. Let $\boldsymbol{X}=\left(X_{1}, \ldots, X_{n}\right)$ and $\boldsymbol{Y}=\left(Y_{1}, \ldots, Y_{n}\right)$ be two $n$-dimensional random vectors. Then $\boldsymbol{X}$ is said to be smaller than $\boldsymbol{Y}$ in the multivariate stochastic order (denoted by $\boldsymbol{X} \leq_{\text {st }} \boldsymbol{Y}$ ) if $\mathrm{E}[\phi(\boldsymbol{X})] \leq \mathrm{E}[\phi(\boldsymbol{Y})]$ for all increasing real-valued functions $\phi$ defined on $\mathbb{R}^{n}$ for which the expectations exist. 
Property 1.1. The random vectors $\boldsymbol{X}$ and $\boldsymbol{Y}$ satisfy $\boldsymbol{X} \leq_{\mathrm{st}} \boldsymbol{Y}$ if and only if there exist two random vectors, $\hat{\boldsymbol{X}}$ and $\hat{\boldsymbol{Y}}$, defined on the same probability space, such that $\boldsymbol{X}={ }_{\text {st }} \hat{\boldsymbol{X}}, \boldsymbol{Y}={ }_{\text {st }} \hat{\boldsymbol{Y}}$, and $\hat{\boldsymbol{X}} \leq \hat{\boldsymbol{Y}}$ a.s.

Finally, the following monotonicity property will be used in Section 2. In the definition, the inequality $\boldsymbol{u} \leq \boldsymbol{v}$, for vectors $\boldsymbol{u}=\left(u_{1}, u_{2}, \ldots, u_{m}\right)$ and $\boldsymbol{v}=\left(v_{1}, v_{2}, \ldots, v_{m}\right)$, means that $u_{i} \leq v_{i}$ for all $i=1,2, \ldots, m$.

Definition 1.4. Let $\left\{\boldsymbol{Y}(\boldsymbol{p}), \boldsymbol{p} \in \mathcal{P} \subseteq \mathbb{R}^{m}, m \in \mathbb{N}\right\}$ be a finite or infinite family of random vectors parametrized by an $m$-dimensional vector of parameters $\boldsymbol{p}$. Then $\{\boldsymbol{Y}(\boldsymbol{p}), \boldsymbol{p} \in \mathcal{P}\}$ is said to be stochastically increasing in $\boldsymbol{p}$ if $\boldsymbol{Y}(\boldsymbol{p}) \leq_{\mathrm{st}} \boldsymbol{Y}\left(\boldsymbol{p}^{\prime}\right)$ for all $\boldsymbol{p} \leq \boldsymbol{p}^{\prime}$.

As we have mentioned above, the purpose of this paper is to study how the variability in time of the environment influences the variability of the populations. To this end, we consider two branching processes defined as in (1.2), but subjected to different random environments, $\boldsymbol{\Theta}_{1}=\left(\Theta_{1,0}, \Theta_{1,1}, \ldots\right)$ and $\boldsymbol{\Theta}_{2}=\left(\Theta_{2,0}, \Theta_{2,1}, \ldots\right)$. Motivated by the comparison results mentioned at the beginning of this section, we derive conditions on the environments in order to ensure that the corresponding populations can be stochastic compared. In particular, we state conditions under which the supermodular order between environments implies the increasing convex order of the populations. Also, we identify conditions under which the concordance order between environments provides comparisons of the expected values of the corresponding populations at every fixed time.

Throughout the following sections, $[X \mid E]$ denotes a random element whose distribution is identical to that of $X$ conditional on the event $E$, and the terms 'increasing' and 'decreasing' are used in the nonstrict sense. Also, for notational convenience, we define $\sum_{j=1}^{0} x_{j}=0$ for every sequence of real numbers $\left\{x_{j}, j \in \mathbb{N}\right\}$.

\section{Comparison results}

Throughout this and the next section, we will make the following assumptions on the array $\boldsymbol{X}(\boldsymbol{\theta})$.

Assumption 2.1. (i) The array $\boldsymbol{X}(\boldsymbol{\theta})$ is an infinite array of nonnegative integer-valued random variables with independent columns of independent variables as described in (1.1).

(ii) For all $k=0,1, \ldots$, the kth column of $\boldsymbol{X}(\boldsymbol{\theta})$ is stochastically increasing in $\theta_{k}$.

(iii) The variables in each column of $\boldsymbol{X}(\boldsymbol{\theta})$ are stochastically increasing, in the sense that $X_{j, k}\left(\theta_{k}\right) \leq_{\text {st }} X_{j+1, k}\left(\theta_{k}\right)$, for all $j, k \in \mathbb{N}$ and $\theta_{k} \in \mathcal{X}$.

Note that, as a particular case, Assumption 2.1(iii) is satisfied when all the variables in each column $\boldsymbol{X}_{k}\left(\theta_{k}\right)$ of $\boldsymbol{X}(\boldsymbol{\theta})$ are i.i.d. for every fixed value of the parameter $\theta_{k}$.

It is easy to verify that, under Assumption 2.1, the $n$th population size, $Z_{n}\left(\theta_{1}, \ldots, \theta_{n}\right)$, is stochastically increasing in $\left(\theta_{1}, \ldots, \theta_{n}\right)$. From this fact, it easily follows that the total population increases in the usual stochastic order as the environment stochastically increases. Actually, using Property 1.1 it is also easy to prove that, always under Assumption 2.1, the whole process $\boldsymbol{Z}\left(\boldsymbol{\Theta}_{1}\right)$ is stochastically smaller than the whole process $\boldsymbol{Z}\left(\boldsymbol{\Theta}_{2}\right)$ (i.e. $\mathrm{E}\left[u\left(\boldsymbol{Z}\left(\boldsymbol{\Theta}_{1}\right)\right)\right] \leq$ $\mathrm{E}\left[u\left(\boldsymbol{Z}\left(\boldsymbol{\Theta}_{2}\right)\right)\right]$ for all increasing functionals $u$ such that both expectations exist) whenever the sequence $\boldsymbol{\Theta}_{1}$ is stochastically smaller than the sequence $\boldsymbol{\Theta}_{2}$.

However, it is natural to imagine that the size of the population at any generation also depends on monotonicity and regularity properties of the environmental process. The following theorem 
is motivated by this observation, and it describes how dependence properties of the process $\Theta$ modify, in increasing convex order sense, the distribution of $Z_{n}\left(\Theta_{1}, \ldots, \Theta_{n}\right)$.

Theorem 2.1. Let $\boldsymbol{X}(\boldsymbol{\theta})$ be an infinite array of nonnegative integer-valued random variables satisfying Assumption 2.1, and let $\Theta_{1}=\left(\Theta_{1,0}, \Theta_{1,1}, \ldots\right)$ and $\Theta_{2}=\left(\Theta_{2,0}, \Theta_{2,1}, \ldots\right)$ be sequences of random variables taking values in $\mathcal{X}$. Assume that both $\boldsymbol{\Theta}_{1}$ and $\boldsymbol{\Theta}_{2}$ are independent of $\boldsymbol{X}(\boldsymbol{\theta})$. Then, for every $n \in \mathbb{N}$, the stochastic inequality

$$
\left(\Theta_{1,0}, \Theta_{1,1}, \ldots, \Theta_{1, n}\right) \leq_{\mathrm{sm}}\left(\Theta_{2,0}, \Theta_{2,1}, \ldots, \Theta_{2, n}\right)
$$

implies that

$$
Z_{n}\left(\Theta_{1,0}, \Theta_{1,1}, \ldots, \Theta_{1, n}\right) \leq_{\text {icx }} Z_{n}\left(\Theta_{2,0}, \Theta_{2,1}, \ldots, \Theta_{2, n}\right) .
$$

Proof. First of all we will prove by induction that, for every fixed $n \in \mathbb{N}$, the function $\phi\left(\theta_{0}, \ldots, \theta_{n}\right)=\mathrm{E}\left[u\left(Z_{n}\left(\theta_{0}, \ldots, \theta_{n}\right)\right)\right]$ is supermodular in $\left(\theta_{0}, \ldots, \theta_{n}\right)$ whenever the function $u$ is increasing and convex.

Since $\phi\left(\theta_{0}, \theta_{1}\right)$ is supermodular by Theorem 2.1 in Belzunce et al. (2006), it is enough to prove that supermodularity of $\phi\left(\theta_{0}, \ldots, \theta_{n}\right)$ in $\left(\theta_{0}, \ldots, \theta_{n}\right)$ follows from supermodularity of $\tilde{\phi}\left(\theta_{0}, \ldots, \theta_{n-1}\right)=\mathrm{E}\left[\tilde{u}\left(Z_{n-1}\left(\theta_{0}, \ldots, \theta_{n-1}\right)\right)\right]$ in $\left(\theta_{0}, \ldots, \theta_{n-1}\right)$ whenever the function $\tilde{u}$ is increasing and convex. To this end, it suffices to show that $\phi\left(\theta_{0}, \ldots, \theta_{n}\right)$ is supermodular in any couple $\left(\theta_{i}, \theta_{k}\right), 0 \leq i<k \leq n$ (see, e.g. Kulik (2003)).

Let us first consider $\left(\theta_{i}, \theta_{n}\right)$ for $0 \leq i<n$. Let $\left(\theta_{i}, \theta_{n}\right)$ and $\left(\theta_{i}^{\prime}, \theta_{n}^{\prime}\right)$ be any two vectors defined on $X^{2}$ such that $\theta_{i} \leq \theta_{i}^{\prime}$ and $\theta_{n} \leq \theta_{n}^{\prime}$. Observe that, since $Z_{n-1}\left(\theta_{1}, \ldots, \theta_{n-1}\right)$ is stochastically increasing in $\left(\theta_{1}, \ldots, \theta_{n-1}\right)$, we can build on the same probability space the random variables $\hat{Z}_{n-1}$ and $\hat{Z}_{n-1}^{\prime}$ such that $\hat{Z}_{n-1}={ }_{\text {st }} Z_{n-1}\left(\theta_{0}, \ldots, \theta_{i}, \ldots, \theta_{n-1}\right), \hat{Z}_{n-1}^{\prime}={ }_{\text {st }}$ $Z_{n-1}\left(\theta_{0}, \ldots, \theta_{i}^{\prime}, \ldots, \theta_{n-1}\right)$, and

$$
\hat{Z}_{n-1} \leq \hat{Z}_{n-1}^{\prime} \quad \text { a.s. }
$$

Thus,

$$
\begin{aligned}
\phi\left(\theta_{0}, \ldots, \theta_{i}^{\prime}, \ldots, \theta_{n}^{\prime}\right)-\phi\left(\theta_{0}, \ldots, \theta_{i}, \ldots, \theta_{n}^{\prime}\right) \\
\quad=\mathrm{E}\left[\mathrm{E}\left[u\left(\sum_{j=1}^{\hat{Z}_{n-1}^{\prime}} X_{j, n}\left(\theta_{n}^{\prime}\right)\right)-u\left(\sum_{j=1}^{\hat{Z}_{n-1}} X_{j, n}\left(\theta_{n}^{\prime}\right)\right) \mid \hat{Z}_{n-1}^{\prime}, \hat{Z}_{n-1}\right]\right] \\
=\mathrm{E}\left[\mathrm{E}\left[g_{\hat{Z}_{n-1}}^{\hat{Z}_{n-1}^{\prime}}\left(\boldsymbol{X}_{n}\left(\theta_{n}^{\prime}\right)\right) \mid \hat{Z}_{n-1}^{\prime}, \hat{Z}_{n-1}\right]\right] \\
\quad \geq \mathrm{E}\left[\mathrm{E}\left[g_{\hat{Z}_{n-1}^{\prime}}\left(\boldsymbol{X}_{n}\left(\theta_{n}\right)\right) \mid \hat{Z}_{n-1}^{\prime}, \hat{Z}_{n-1}\right]\right] \\
=\mathrm{E}\left[\mathrm{E}\left[u\left(\sum_{j=1}^{\hat{Z}_{n-1}^{\prime}} X_{j, n}\left(\theta_{n}\right)\right)-u\left(\sum_{j=1}^{\hat{Z}_{n-1}} X_{j, n}\left(\theta_{n}\right)\right) \mid \hat{Z}_{n-1}^{\prime}, \hat{Z}_{n-1}\right]\right] \\
=\phi\left(\theta_{0}, \ldots, \theta_{i}^{\prime}, \ldots, \theta_{n}\right)-\phi\left(\theta_{0}, \ldots, \theta_{i}, \ldots, \theta_{n}\right),
\end{aligned}
$$

where the inequality follows from (2.1), Assumption 2.1(ii), and the fact that the function $g_{l}^{m}(\bar{y})=u\left(\sum_{i=1}^{m} y_{i}\right)-u\left(\sum_{i=1}^{l} y_{i}\right)$ is an increasing function of $\bar{y}=\left\{y_{1}, y_{2}, \ldots\right\}$ whenever 
$m \geq l$ and $\bar{y}$ is any sequence of nonnegative integers. Thus, clearly, we obtain

$$
\begin{aligned}
& \phi\left(\theta_{0}, \ldots, \theta_{i}^{\prime}, \ldots, \theta_{n}^{\prime}\right)+\phi\left(\theta_{0}, \ldots, \theta_{i}, \ldots, \theta_{n}\right) \\
& \quad \geq \phi\left(\theta_{0}, \ldots, \theta_{i}, \ldots, \theta_{n}^{\prime}\right)+\phi\left(\theta_{0}, \ldots, \theta_{i}^{\prime}, \ldots, \theta_{n}\right),
\end{aligned}
$$

i.e. $\phi\left(\theta_{0}, \ldots, \theta_{n}\right)$ is supermodular in $\left(\theta_{i}, \theta_{n}\right), 0 \leq i<n$.

Now we consider $\left(\theta_{i}, \theta_{k}\right)$ for $0 \leq i<k<n$. Observe that the function $\tilde{u}(z)=$ $u\left(\sum_{j=1}^{z} y_{j}\right)$ is increasing and convex in $z \in \mathbb{N}$ whenever $u$ is an increasing and convex function and $\bar{y}=\left\{y_{1}, y_{2}, \ldots\right\}$ is any increasing sequence of nonnegative integers. Also, recall that, by the inductive assumption, the function $\tilde{\phi}\left(\theta_{0}, \ldots, \theta_{n-1}\right)=\mathrm{E}\left[\tilde{u}\left(Z_{n-1}\left(\theta_{0}, \ldots, \theta_{n-1}\right)\right)\right]$ is supermodular in $\left(\theta_{0}, \ldots, \theta_{n-1}\right)$ for every increasing and convex function $\tilde{u}$. Moreover, by Assumption 2.1(iii) we can build on the same probability space the random sequence $\hat{\boldsymbol{X}}_{n}=\left\{\hat{X}_{j, n}, j \in \mathbb{N}\right\}$ such that $\hat{X}_{j, n}\left(\theta_{n}\right)=_{\text {st }} X_{j, n}\left(\theta_{n}\right)$ and

$$
\hat{X}_{j, n}\left(\theta_{n}\right) \leq \hat{X}_{j+1, n}\left(\theta_{n}\right) \quad \text { a.s. }
$$

for all $j, n \in \mathbb{N}$. Thus, letting $\hat{\boldsymbol{X}}_{n}\left(\theta_{n}\right)=\left\{\hat{X}_{j, n}\left(\theta_{n}\right), j \in \mathbb{N}\right\}$, we obtain

$$
\begin{gathered}
\phi\left(\theta_{0}, \ldots, \theta_{i}^{\prime}, \ldots, \theta_{k}^{\prime}, \ldots, \theta_{n}\right)-\phi\left(\theta_{0}, \ldots, \theta_{i}, \ldots, \theta_{k}^{\prime}, \ldots, \theta_{n}\right) \\
=\mathrm{E}\left[\mathrm { E } \left[u\left(\sum_{j=1}^{Z_{n-1}\left(\theta_{0}, \ldots, \theta_{i}^{\prime}, \ldots, \theta_{k}^{\prime}, \ldots, \theta_{n}\right)} \hat{X}_{j, n}\left(\theta_{n}\right)\right)\right.\right. \\
\left.\left.\quad-u\left(\sum_{j=1}^{Z_{n-1}\left(\theta_{0}, \ldots, \theta_{i}, \ldots, \theta_{k}^{\prime}, \ldots, \theta_{n}\right)} \hat{X}_{j, n}\left(\theta_{n}\right)\right) \mid \hat{\boldsymbol{X}}_{n}\left(\theta_{n}\right)\right]\right] \\
=\mathrm{E}\left[\mathrm { E } \left[\tilde{u}\left(Z_{n-1}\left(\theta_{0}, \ldots, \theta_{i}^{\prime}, \ldots, \theta_{k}^{\prime}, \ldots, \theta_{n}\right)\right)\right.\right. \\
\left.\left.\quad-\tilde{u}\left(Z_{n-1}\left(\theta_{0}, \ldots, \theta_{i}, \ldots, \theta_{k}^{\prime}, \ldots, \theta_{n}\right)\right) \mid \hat{\boldsymbol{X}}_{n}\left(\theta_{n}\right)\right]\right] \\
\geq \mathrm{E}\left[\mathrm { E } \left[\tilde{u}\left(Z_{n-1}\left(\theta_{0}, \ldots, \theta_{i}^{\prime}, \ldots, \theta_{k}, \ldots, \theta_{n}\right)\right)\right.\right. \\
\left.\left.\quad-\tilde{u}\left(Z_{n-1}\left(\theta_{0}, \ldots, \theta_{i}, \ldots, \theta_{k}, \ldots, \theta_{n}\right)\right) \mid \hat{\boldsymbol{X}}_{n}\left(\theta_{n}\right)\right]\right] \\
=\mathrm{E}\left[\mathrm { E } \left[u\left(\sum_{j=1}^{Z_{n-1}\left(\theta_{0}, \ldots, \theta_{i}^{\prime}, \ldots, \theta_{k}, \ldots, \theta_{n}\right)} \hat{X}_{j, n}\left(\theta_{n}\right)\right)\right.\right. \\
\left.\left.\quad-u\left(\sum_{j=1}^{Z_{n-1}\left(\theta_{0}, \ldots, \theta_{i}, \ldots, \theta_{k}, \ldots, \theta_{n}\right)} \hat{X}_{j, n}\left(\theta_{n}\right)\right) \mid \hat{\boldsymbol{X}}_{n}\left(\theta_{n}\right)\right]\right] \\
=\phi\left(\theta_{0}, \ldots, \theta_{i}^{\prime}, \ldots, \theta_{k}, \ldots, \theta_{n}\right)-\phi\left(\theta_{0}, \ldots, \theta_{i}, \ldots, \theta_{k}, \ldots, \theta_{n}\right),
\end{gathered}
$$

where the inequality follows from remarks above on the function $\tilde{u}$, inequality (2.2), and the subsequent supermodularity of $\mathrm{E}\left[\tilde{u}\left(Z_{n-1}\left(\theta_{0}, \ldots, \theta_{n-1}\right)\right) \mid \hat{\boldsymbol{X}}_{n}\left(\theta_{n}\right)\right]$.

Thus, $\phi\left(\theta_{0}, \ldots, \theta_{n}\right)$ is also supermodular in $\left(\theta_{i}, \theta_{k}\right), 0 \leq i<k<n$, and supermodularity of $\phi\left(\theta_{0}, \ldots, \theta_{n}\right)$ in $\left(\theta_{0}, \ldots, \theta_{n}\right)$ follows.

Now we get the increasing convex comparison between population sizes $Z_{n}\left(\Theta_{1,0}, \Theta_{1,1}, \ldots\right.$, $\left.\Theta_{1, n}\right)$ and $Z_{n}\left(\Theta_{2,0}, \Theta_{2,1}, \ldots, \Theta_{2, n}\right)$ just by observing that, for every fixed increasing and 
convex function $u$,

$$
\begin{aligned}
\mathrm{E}\left[u\left(Z_{n}\left(\Theta_{1,0}, \Theta_{1,1}, \ldots, \Theta_{1, n}\right)\right)\right] & =\mathrm{E}\left[\phi\left(\Theta_{1,0}, \Theta_{1,1}, \ldots, \Theta_{1, n}\right)\right] \\
& \leq \mathrm{E}\left[\phi\left(\Theta_{2,0}, \Theta_{2,1}, \ldots, \Theta_{2, n}\right)\right] \\
& =\mathrm{E}\left[u\left(Z_{n}\left(\Theta_{2,0}, \Theta_{2,1}, \ldots, \Theta_{2, n}\right)\right)\right]
\end{aligned}
$$

holds, where the function $\phi$ is defined as above.

Under weaker assumptions, we can also obtain weaker comparisons between the expected margins of the two branching processes, as stated in the following result.

Theorem 2.2. Let $\boldsymbol{X}(\boldsymbol{\theta})$ satisfy Assumption 2.1(i), and let $\boldsymbol{\Theta}_{1}=\left(\Theta_{1,0}, \Theta_{1,1}, \ldots\right)$ and $\boldsymbol{\Theta}_{2}=$ $\left(\Theta_{2,0}, \Theta_{2,1}, \ldots\right)$ be sequences of random variables taking values in $\mathcal{X}$. Assume that both $\Theta_{1}$ and $\boldsymbol{\Theta}_{2}$ are independent of $\boldsymbol{X}(\boldsymbol{\theta})$, and that $\mathrm{E}\left[X_{1, k}\left(\theta_{k}\right)\right]$ is increasing in $\theta_{k}$ for all $k=0,1, \ldots$ Then, for every $n \in \mathbb{N}$, the stochastic inequality

$$
\left(\Theta_{1,0}, \Theta_{1,1}, \ldots, \Theta_{1, n}\right) \leq_{c}\left(\Theta_{2,0}, \Theta_{2,1}, \ldots, \Theta_{2, n}\right)
$$

implies that

$$
\mathrm{E}\left[Z_{n}\left(\Theta_{1,0}, \Theta_{1,1}, \ldots, \Theta_{1, n}\right)\right] \leq \mathrm{E}\left[Z_{n}\left(\Theta_{2,0}, \Theta_{2,1}, \ldots, \Theta_{2, n}\right)\right]
$$

Proof. Let $\left(\theta_{0}, \ldots, \theta_{n}\right) \in X^{n+1}$ and note that $\mathrm{E}\left[Z\left(\theta_{0}, \ldots, \theta_{n}\right)\right]=\prod_{k=0}^{n} \mathrm{E}\left[X_{1, k}\left(\theta_{k}\right)\right]$ (the equality trivially comes from the fact that all the random variables $X_{j, k}\left(\theta_{k}\right)$ are i.i.d. for fixed values of $k$ ). Observing that, by assumption, every $\mathrm{E}\left[X_{1, k}\left(\theta_{k}\right)\right]$ is increasing in $\theta_{k}$, by (2.3) we obtain

$$
\begin{aligned}
\mathrm{E}\left[Z\left(\Theta_{1,0}, \ldots, \Theta_{1, n}\right)\right] & =\mathrm{E}\left[\mathrm{E}\left[Z_{n}\left(\Theta_{1,0}, \ldots, \Theta_{1, n}\right) \mid\left(\Theta_{1,0}, \ldots, \Theta_{1, n}\right)\right]\right] \\
& =\mathrm{E}\left[\prod_{k=0}^{n} \mathrm{E}\left[X_{1, k}\left(\Theta_{1, k}\right)\right]\right] \\
& \leq \mathrm{E}\left[\prod_{k=0}^{n} \mathrm{E}\left[X_{1, k}\left(\Theta_{2, k}\right)\right]\right] \\
& =\mathrm{E}\left[\mathrm{E}\left[Z_{n}\left(\Theta_{2,0}, \ldots, \Theta_{2, n}\right) \mid\left(\Theta_{2,0}, \ldots, \Theta_{2, n}\right)\right]\right] \\
& =\mathrm{E}\left[Z\left(\Theta_{2,0}, \ldots, \Theta_{2, n}\right)\right],
\end{aligned}
$$

i.e. the assertion.

\section{An example of an application}

Assume that the random evolutions of the environment are described by a stationary discretetime homogeneous Markov process $\Theta=\left\{\Theta_{n}, n \in \mathbb{N}\right\}$ that is stochastically monotone (i.e. such that $\left[\Theta_{2} \mid \Theta_{1}=\theta\right]$ is stochastically increasing in $\theta$ ). Using the criteria described in Section 2, we can define stochastic bounds for the total population at any generation. In fact, let $\boldsymbol{\Theta}_{1}=$ $\left\{\Theta_{1, n}, n \in \mathbb{N}\right\}$ be a sequence of variables such that $\Theta_{1, n}=\Theta_{1,0}$ a.s. for all $n \in \mathbb{N}$, where $\Theta_{1,0}$ has the same distribution as $\Theta_{0}$ (i.e. the stationary marginal distribution of $\boldsymbol{\Theta}$ ). Then it is well known that $\left(\Theta_{0}, \Theta_{1}, \ldots, \Theta_{n}\right) \leq \leq_{\mathrm{sm}}\left(\Theta_{1,0}, \Theta_{1,1}, \ldots, \Theta_{1, n}\right)$, for every $n \in \mathbb{N}$ (see, e.g. Tchen (1980)). 
Now let $\boldsymbol{\Theta}_{2}=\left\{\Theta_{2, n}, n \in \mathbb{N}\right\}$ be a sequence of i.i.d. variables such that $\Theta_{2, n}={ }_{\text {st }} \Theta_{0}$ (i.e. having the same distribution as the stationary marginal distribution of $\Theta$ ). It has been shown (see, e.g. Hu and Pan (2000)) that, in this case, $\left(\Theta_{2,0}, \Theta_{2,1}, \ldots, \Theta_{2, n}\right) \leq$ sm $\left(\Theta_{0}, \Theta_{1}, \ldots, \Theta_{n}\right)$ holds, for every $n \in \mathbb{N}$.

Therefore, for the branching process $\boldsymbol{Z}(\boldsymbol{\Theta})$ defined as in (1.2), and subjected to an underlying stationary discrete-time homogeneous Markov process $\boldsymbol{\Theta}$, the following two assertions hold.

Corollary 3.1. Let $\boldsymbol{X}(\boldsymbol{\theta})$ be an infinite array of nonnegative integer-valued random variables satisfying Assumption 2.1. If $\boldsymbol{X}(\boldsymbol{\theta})$ is independent of $\boldsymbol{\Theta}$ then we obtain

$$
Z_{n}\left(\Theta_{2,0}, \Theta_{2,1}, \ldots, \Theta_{2, n}\right) \leq_{\text {icx }} Z_{n}\left(\Theta_{0}, \Theta_{1}, \ldots, \Theta_{n}\right) \leq_{\text {icx }} Z_{n}\left(\Theta_{1,0}, \Theta_{1,1}, \ldots, \Theta_{1, n}\right),
$$

for every $n \in \mathbb{N}$.

Corollary 3.2. Let $\boldsymbol{X}(\boldsymbol{\theta})$ be an infinite array of nonnegative integer-valued random variables satisfying Assumption 2.1(i). If $\mathrm{E}\left[X_{1, k}\left(\theta_{k}\right)\right]$ is increasing in $\theta_{k}$, for all $k=0,1, \ldots$, and if $\boldsymbol{X}(\boldsymbol{\theta})$ is independent of $\boldsymbol{\Theta}$, then

$$
\mathrm{E}\left[Z_{n}\left(\Theta_{2,0}, \Theta_{2,1}, \ldots, \Theta_{2, n}\right)\right] \leq \mathrm{E}\left[Z_{n}\left(\Theta_{0}, \Theta_{1}, \ldots, \Theta_{n}\right)\right] \leq \mathrm{E}\left[Z_{n}\left(\Theta_{1,0}, \Theta_{1,1}, \ldots, \Theta_{1, n}\right)\right],
$$

for every $n \in \mathbb{N}$.

The interest in these results is due to the fact that the distributions of $Z_{n}\left(\Theta_{2,0}, \Theta_{2,1}, \ldots\right.$, $\left.\Theta_{2, n}\right)$ and $Z_{n}\left(\Theta_{1,0}, \Theta_{1,1}, \ldots, \Theta_{1, n}\right)$ can be calculated in closed form by observing that these two processes are nothing else than a standard branching process and a mixture of standard branching processes. Note also that if $\boldsymbol{\Theta}$ describes the behavior of the environment and the columns of $\boldsymbol{X}(\boldsymbol{\theta})$ are stochastically increasing in the parameters $\theta_{k}$, then the assumption that $\boldsymbol{\Theta}$ is stochastically monotone is realistic and common in applicative contexts.

Always assuming that the underlying process $\boldsymbol{\Theta}$ is a stationary discrete-time homogeneous Markov process, other interesting examples of the application of the results presented in Section 2 may be provided by considering Theorem 3.2 and Theorem 4.1 of $\mathrm{Hu}$ and Pan (2000).

\section{Acknowledgements}

I would like to thank Professor Moshe Shaked and an anonymous referee for their valuable comments and suggestions that greatly improved the presentation of this paper. This research was partially supported by Italian PRIN-Cofin 2004 Stochastic Models in Mathematical Finance.

\section{References}

Athreya, K. B. And Karlin, S. (1970). On branching processes with random environments. Bull. Amer. Math. Soc. 76, 865-870.

BäUerle, N. And Rieder, U. (1997). Comparison results for Markov-modulated recursive models. Prob. Eng. Inf. Sci. 11, 203-217.

Belzunce, F., Ortega, E., Pellerey, F. And Ruiz, J. M. (2006). Variability of total claim amounts under dependence between claims severity and number of claims. Insurance Math. Econom. 38, 460-468.

Harris, T. E. (1989). The Theory of Branching Processes. Dover, New York.

Hu, T. and Pan, X. (2000). Comparisons of dependence for stationary Markov processes. Prob. Eng. Inf. Sci. 14, 299-315.

JAGERS, P. AND Lu, Z. (2002). Branching processes with deteriorating random environments. J. Appl. Prob. 39, 395-401.

JoE, H. (1997). Multivariate Models and Dependence Concepts. Chapman \& Hall, London. 
Kimmel, M. And Axelrod, D. E. (2002). Branching Processes in Biology. Springer, New York.

KULIK, R. (2003). Stochastic comparisons of multivariate random sums. Appl. Math. 30, 379-387.

Müller, A. AND SCARsini, M. (2000). Some remarks on the supermodular order. J. Multivariate Anal. 73, $107-119$.

Rolski, T., Schmidli, H., Schmidt, V. AND Teugels, J. (1999). Stochastic Processes for Insurance and Finance. John Wiley, New York.

Ross, S. M. (1983). Stochastic Processes. John Wiley, New York.

Shaked, M. And Shanthikumar, J. G. (1994). Stochastic Orders and Their Applications. Academic Press, New York.

Shaked, M. AND Shanthikumar, J. G. (1997). Supermodular stochastic orders and positive dependence of random vectors. J. Multivariate Anal. 61, 86-101.

Smith, W. L. ANd WiLkinson, W. E. (1969). On branching processes in random environments. Ann. Math. Statist. 40, 814-827.

TCHen, A. H. (1980). Inequalities for distributions with given marginals. Ann. Prob. 8, 814-827.

Teich, M. C. And Saleh, B. E. A. (2000). Branching processes in quantum electronics. IEEE J. Sel. Topics Quantum Electron. 6, 1450-1457. 